\title{
Effect of Anti-Siglec-F Antibody and Reactive Oxygen Species Blocking on Histamine Release in Urinary Bladder of Ovalbumin- Treated Mice
}

\author{
Bo-Hwa Choi ${ }^{\star}$, Gwoan-Youb Choo ${ }^{1, \star}$, Ju-Hee Kang, Choong-Yeol Lee ${ }^{2}$, Chang-Shin Park \\ Departments of Pharmacology and ${ }^{1}$ Urology, Inha University School of Medicine, BK 21 Project, Incheon; \\ ${ }^{2}$ Department of Oriental Physiology, Gachon University College of Oriental Medicine, Seongnam, Korea
}

\begin{abstract}
Purpose: Sialic acid-binding Ig-like lectin (Siglec) is an immune inhibitory receptor that plays a role in the negative regulation of the activation of immune cells. This study aimed to evaluate the effects of anti-Siglec-F on plasma and urinary histamine levels in ovalbumin (OVA)-challenged urinary bladder in mice.

Methods: Thirty BALB/c mice were used. In group I (control group, $n=5$ ), mice were sensitized with OVA and challenged with saline. In group II (OVA challenge group, $n=5$ ), OVA was used for intraperitoneal sensitization and intravesical challenge. The challenged mice in group III (control immunoglobulin G [IgG] group, $n=5$ ) and those in group IV (anti-Siglec-F group, $n=5$ ) were intraperitoneally pretreated with rabbit control IgG or anti-Siglec-F antibody, respectively. In groups $\mathrm{V}$ ( $\mathrm{N}$-acetylcysteine [NAC] in OVA challenge group, $n=5$ ) and VI (control NAC only, $n=5$ ), mice were pretreated with NAC.

Results: Urinary histamine concentrations were significantly higher 7 days after intravesical OVA challenge $(\mathrm{P}<0.01)$, whereas plasma histamine levels were not. Pretreatment with anti-Siglec-F antibody significantly prevented the increase in urinary histamine release $(\mathrm{P}<0.05)$, whereas pretreatment with the IgG antibody control did not. Also, pretreatment of the OVA challenge group with NAC did not affect the histamine concentration in either urine or plasma.

Conclusions: Systemic anti-Siglec-F treatment showed anti-allergic effects at least on local histamine release, particularly in the lower urinary bladder.
\end{abstract}

Keywords: Histamine; Mice; Ovalbumin; Reactive oxygen species; Sialic acid binding Ig-like lectin; Urinary bladder

\section{INTRODUCTION}

Sialic acid-binding Ig-like lectins (Siglecs) are members of an immunoglobulin receptor superfamily with extracellular and cytoplasmic domains. In general, the extracellular domain binds to several forms of sialic acid, and the cytoplasmic domain has characteristics of immunoreceptor tyrosine-based inhibitory motifs, which are known to have an inhibitory signaling activity in immune pathways [1].

In the murine allergy model, Siglec-F belongs to the CD33related Siglec family and is selectively expressed in eosinophils and is considered to be the functional ortholog of human Siglec-8 [2]. Siglec-F is also expressed on alveolar macrophages, bone marrow-derived dendritic cells, and bone marrow-derived macrophages with differential levels [3]. Activation of Siglec-F can induce apoptosis of eosinophils and ameliorate allergen-induced eosinophilic inflammation [4]. Siglec-F knockout mice show a more prominent eosinophilia in bone marrow, peripheral blood, and lung tissue after allergen sensitization and challenge [5].

Recently, we discussed in a short review that the chronic bladder inflammatory disease, interstitial cystitis, can be induced by
Corresponding author: Chang-Shin Park

Department of Pharmacology, Inha University School of Medicine, 5th Floor, C-Dong, 366 Seohae-daero, Jung-gu, Incheon 400-103, Korea

Tel: +82-32-890-0962 / Fax: +82-32-887-7488 / E-mail: parkshin@inha.ac.kr

${ }^{\star}$ These authors contributed equally to this study.

Submitted: September 15, 2012 / Accepted after revision: September 28, 2012
This is an Open Access article distributed under the terms of the Creative Commons Attribution Non-Commercial License (http://creativecommons.org/licenses/by-nc/3.0/) which permits unrestricted non-commercial use, distribution, and reproduction in any medium, provided the original work is properly cited. 
localized allergic reactions and is mediated mainly through the activation of infiltrated mast cells with involvement of reactive oxygen species (ROS). Thus, a possible hypothesis was suggested for utilization of Siglec-specific monoclonal antibodies or their glycan ligands as a therapeutic signal to induce inhibitory signaling activity with ROS dependency [6]. However, there have still been no reports of the application of anti-Siglec-F antibody on local allergy in urinary bladder.

Therefore, we aimed to evaluate the therapeutic effect of antiSiglec-F antibody or ROS blocking in a murine model of ovalbumin (OVA)-sensitized and challenged bladder allergy, particularly by assessing histamine release in plasma and urine.

\section{MATERIALS AND METHODS}

\section{Animals}

Female BALB/c mice were purchased from Orient Bio (Seongnam, Korea) and were used at 8 weeks of age. All procedures of the animal protocol in our study were performed in accordance with the Guide for the Care and Use of Laboratory Animals of the National Institutes of Health and were approved by the Inha University Institutional Animal Care and Use Committee on ethics.

\section{Surgical Catheter Implantations}

Catheter implantations were performed as previously described [7]. Briefly, mice were anesthetized with ketamine (Ketamine, $75 \mathrm{mg} / \mathrm{kg}$, i.p.; Yuhan, Seoul, Korea) and xylazine (Rompun, 15 mg/kg, i.p.; Bayer Korea Ltd., Seoul, Korea). Through the lower abdominal incision, a polyethylene catheter (PE-10, BD, Franklin Lakes, NJ, USA) with a cuff was inserted into the dome of the bladder to inject vehicle or OVA. The catheters were tunneled subcutaneously and anchored to the skin of the back with a silk ligature. The free ends of the catheters were sealed.

\section{Antigen Sensitization and Challenge}

The sensitization and antigen challenge for the murine model was performed as previously described with slight modification $[8,9]$. For OVA sensitization, animals were divided into six groups. Under pathogen-free conditions, OVA (40 $\mu \mathrm{g} / \mathrm{kg}$; SigmaAldrich Co., St. Louis, MO, USA) diluted in $0.1 \mathrm{~mL}$ saline was given by intraperitoneal injection with aluminum hydroxide gel (alum adjuvant, $40 \mathrm{mg} / \mathrm{kg}$ ) four times on days 1, 5, 14, and 21 as applied in allergy experiments [8]. For repeated OVA challenge, from the day after the last sensitization, $0.1 \mathrm{~mL}$ of OVA
(10 mg) or the vehicle control (saline) was daily and intravesically injected via catheter to unanesthetized animals for 7 days. Twenty-four hours after the last OVA challenge, blood and urine were collected.

\section{Treatment of Anti-Siglec-F and $\mathrm{N}$-acetylcysteine (NAC)}

Mouse anti-Siglec-F antibody (Monoclonal Rat $\operatorname{IgG}_{2 \mathrm{~A}}$ clone \#238047, R\&D Systems, Minneapolis, MN, USA) was used for the experiments. Anti-Siglec-F (10 $\mu \mathrm{g} /$ mouse) was given by intraperitoneal injection 1 hour before OVA intravesical challenge on days $22,23,24,25,26,27$, and 28 . In the control group, rabbit control immunoglobulin G (IgG) (purified normal rabbit IgG, R\&D Systems) was intraperitoneally injected by the same dose and schedule. In this study, we also compared the effects of ROS blocking by pretreatment with NAC.

In group I (control group, $n=5$ ), mice were sensitized with OVA and challenged with saline. In group II (OVA challenge group, $n=5$ ), OVA was used for intraperitoneal sensitization and intravesical challenge. Mice in group III (control IgG group, $\mathrm{n}=5$ ) and those in group IV (anti-Siglec-F group, $\mathrm{n}=5$ ) were pretreated before OVA challenge with rabbit control IgG and anti-Siglec-F antibody by intraperitoneal injection, respectively. Mice in group V (NAC-group, $\mathrm{n}=5$ ) and those in group VI (control NAC only group, $n=5$ ) were pretreated with saline or NAC by intraperitoneal injection, respectively, before every OVA challenge.

\section{Plasma and Urinary Histamine Measurements}

Aortic puncture was performed for collecting blood after sacrifice. To measure plasma histamine concentrations, blood was collected into ethylenediaminetetraacetic acid tubes on ice and plasma was isolated by centrifugation. Perchloride acid $(475 \mu \mathrm{L}$ of $0.4 \mathrm{M}$ ) was added to $25 \mu \mathrm{L}$ of each plasma sample. Histamine was assayed spectrophotofluorimetrically after condensation with $o$-phthaldialdehyde as described [10]. Urinary histamine concentrations were also measured by the same method.

\section{Statistical Analyses}

Levels of plasma and urinary histamine were compared between groups by using unpaired $t$-tests. Values of $\mathrm{P}<0.05$ or $<0.01$ were considered to be statistically significant.

\section{RESULTS}

As shown in Fig. 1, urinary histamine concentrations were sig- 

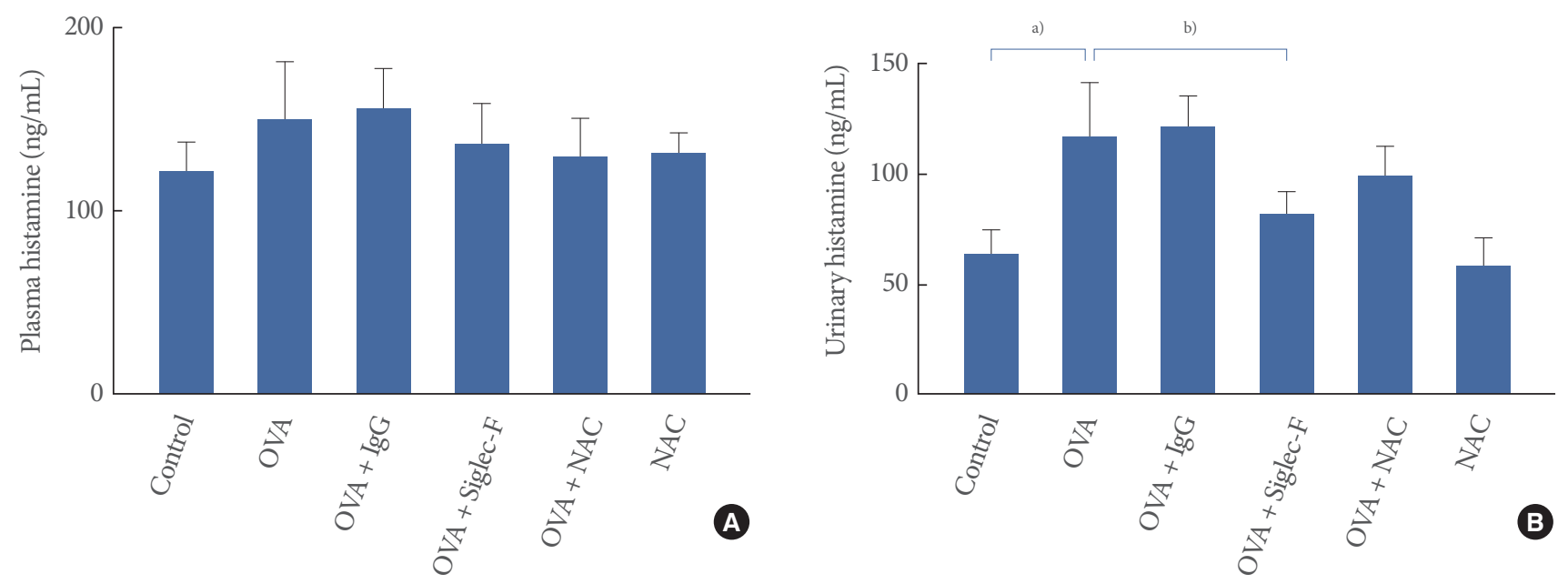

Fig. 1. Histamine concentrations. Histamine release was measured 7 days after ovalbumin (OVA) challenge and pretreatment with Siglec-F antibody or $\mathrm{N}$-acetylcysteine (NAC) from isolated plasma (A) and urine (B). Data are presented as mean $\pm \mathrm{SD}$. Control, OVAsensitized and saline-challenged (group I); OVA, OVA-sensitized and OVA-challenged (group II); OVA+IgG, OVA-sensitized, OVAchallenged, and immunoglobulin G (IgG)-pre-treated (group III); OVA+Siglec-F, OVA-sensitized, OVA-challenged, and anti-SiglecF-pretreated (group IV); OVA+NAC, OVA-sensitized, OVA-challenged, and NAC-pretreated (group V); NAC, OVA-sensitized and NAC-treated (group VI) ${ }^{\text {a) }} \mathrm{P}<0.01$ and ${ }^{\text {b) }} \mathrm{P}<0.05$ : significant difference with Control or OVA group.

nificantly higher 7 days after intravesical OVA challenge (group II) compared with the control group I $(\mathrm{P}<0.01)$, but plasma histamine levels were not. Anti-Siglec-F treatment before every intravesical OVA challenge for 7 days significantly prevented the increase in intravesically OVA-challenged histamine release in urine $(\mathrm{P}<0.05)$, whereas pretreatment with the IgG antibody control did not (Fig. 1). There were no changes in plasma histamine levels in the anti-Siglec-F- or the IgG-treated groups (Fig. 1).

We also examined the effects of ROS blocking by pretreatment with $10 \mathrm{mM}$ NAC on the OVA-induced histamine release. Unexpectedly, there were no significant effects of pretreatment with NAC on the OVA-challenged histamine release in either urine or plasma (Fig. 1).

\section{DISCUSSION}

This study demonstrates first that pretreatment with an anti-Siglec-F antibody to mice intravesically challenged with OVA to induce bladder allergy decreased the urine levels of histamine release, but not the levels in plasma. However, we have no evidence concerning cell-mediated mechanisms, such as apoptosis of eosinophils or inactivation of mast cells.

There are few reports of the expression of Siglecs in rats; thus, the available anti-Siglec-F antibody of the murine type was used for this study. However, murine mast cells do not express Siglec$\mathrm{F}$ [11]. Siglec-F is considered to be the functional ortholog of human Siglec-8, which is expressed selectively in eosinophils, mast cells, and basophils [2,12-15]. Additionally, we have successfully performed an experiment for therapeutic applications of Siglec-F to local airway allergy challenged with OVA in a murine model, although without results for the activation of mast cells [8].

Ahluwalia et al. [9] established a bladder allergy model by local challenge with OVA in OVA-sensitized rats. Our experiment was designed with some modifications on the basis of the allergy model methods established by Kim et al. [8] and Ahluwalia et al. [9]. Interestingly, local histamine release significantly increased in urine in mice intravesically challenged with OVA, and pretreatment with Siglec-F significantly prevented the increase. However, we have no data on the degranulation of mast cells. Considering this point, we suggest that the effect of systemic anti-Siglec-F on murine mast cells and their release of histamine could be minimal, because of selectivity for expression of Siglec-F, particularly in the murine model. Additionally, in this study, we did not completely explain the involvement of ROS, because there was no effect on histamine release of ROS blocking with NAC.

Our results imply that systemic regulation of histamine release with anti-Sigelc-F antibody could be effective in local al- 
lergen challenge after systemic sensitization. Thus, Siglec-F could be considered a future therapeutic target for treating allergic or inflammatory reactions in urinary bladder, such as interstitial cystitis.

In conclusion, we suggest that anti-Siglec-F treatment may have an effect on controlling the histamine release from the urinary bladder in an experimental allergic model.

\section{CONFLICT OF INTEREST}

No potential conflict of interest relevant to this article was reported.

\section{ACKNOWLEDGEMENTS}

This study was also supported by a research fund (E00004) from the National Research Foundation of Korea.

\section{REFERENCES}

1. Bochner BS. Siglec-8 on human eosinophils and mast cells, and Siglec-F on murine eosinophils, are functionally related inhibitory receptors. Clin Exp Allergy 2009;39:317-24.

2. O'Reilly MK, Paulson JC. Siglecs as targets for therapy in immunecell-mediated disease. Trends Pharmacol Sci 2009;30:240-8.

3. Tateno H, Li H, Schur MJ, Bovin N, Crocker PR, Wakarchuk WW, et al. Distinct endocytic mechanisms of CD22 (Siglec-2) and SiglecF reflect roles in cell signaling and innate immunity. Mol Cell Biol 2007;27:5699-710.

4. Song DJ, Cho JY, Lee SY, Miller M, Rosenthal P, Soroosh P, et al. Anti-Siglec-F antibody reduces allergen-induced eosinophilic inflammation and airway remodeling. J Immunol 2009;183:5333-41.

5. Zhang M, Angata T, Cho JY, Miller M, Broide DH, Varki A. Defining the in vivo function of Siglec-F, a CD33-related Siglec expressed on mouse eosinophils. Blood 2007;109:4280-7.
6. Park CS, Bochner BS. Potential targeting of siglecs, mast cell inhibitory receptors, in interstitial cystitis. Int Neurourol J 2011;15:61-3.

7. Jin LH, Han JU, Park CS, Shin HY, Yoon SM, Lee T. Intravesical PGE2 administration in conscious rats as an experimental model of detrusor overactivity observed by simultaneous registrations of intravesical and intraabdominal pressures. Int Neurourol J 2010; 14:69-77.

8. Kim YH, Yang TY, Park CS, Ahn SH, Son BK, Kim JH, et al. AntiIL-33 antibody has a therapeutic effect in a murine model of allergic rhinitis. Allergy 2012;67:183-90.

9. Ahluwalia A, Giuliani S, Scotland R, Maggi CA. Ovalbumin-induced neurogenic inflammation in the bladder of sensitized rats. Br J Pharmacol 1998;124:190-6.

10. Bergstrand H, Lundquist B. Human basophil histamine release is differently affected by inhibitors of calmodulin, diacylglycerol kinase and peptidyl prolyl cis-trans isomerase in a secretagogue specific manner. Allergy 1992;47(4 Pt 2):353-61.

11. Tateno H, Crocker PR, Paulson JC. Mouse Siglec-F and human Siglec-8 are functionally convergent paralogs that are selectively expressed on eosinophils and recognize 6-sulfo-sialyl Lewis X as a preferred glycan ligand. Glycobiology 2005;15:1125-35.

12. Song DJ, Cho JY, Miller M, Strangman W, Zhang M, Varki A, et al. Anti-Siglec-F antibody inhibits oral egg allergen induced intestinal eosinophilic inflammation in a mouse model. Clin Immunol 2009; 131:157-69.

13. Crocker PR, Paulson JC, Varki A. Siglecs and their roles in the immune system. Nat Rev Immunol 2007;7:255-66.

14. Yokoi H, Myers A, Matsumoto K, Crocker PR, Saito H, Bochner BS. Alteration and acquisition of Siglecs during in vitro maturation of CD34+ progenitors into human mast cells. Allergy 2006;61:76976.

15. Kiwamoto T, Kawasaki N, Paulson JC, Bochner BS. Siglec-8 as a drugable target to treat eosinophil and mast cell-associated conditions. Pharmacol Ther 2012;135:327-36. 